Apidologie, 1986, 17 (2), 175-192

\title{
DIE ABLAGERUNG VON KÖNIGINNENFUTTERSAFT UND DAS WACHSTUM VON KÖNIGINNENLARVEN BEI WIEDERHOLTER PFLEGE IM WEISELLOSEN VOLK VON APIS MELLIFICA L.
}

\author{
Karl WEISS \\ Bayerische Landesanstalt für Bienenzucht \\ Burgbergstraße 70, 8520 Erlangen
}

\section{ZUSAMMENFASSUNG}

Bei mehrmals aufeinanderfolgenden Pflegeansätzen in weisellosen Völkern wurden Beobachtungen über Menge und Zeitabfolge der Futtersaftablagerung, über das Wachstum der Königinnenlarven im alternden Pflegevolk, über die fraglichen Abhängigkeiten von Futtersaftmenge und Larvengewicht sowie Futtersaftmenge und «Umlarvalter» gemacht. Außerdem wurden Wachs- und Kunststoffnäpfchen im Hinblick auf die Versorgung ihrer Insassen mit Weiselfuttersaft verglichen. In der Diskussion wurden praktische Aspekte der Futtersaftgewinnung berücksichtigt.

\section{EINLEITUNG}

Es gibt in der Literatur eine Reihe von Arbeiten über die kommerzielle Gewinnung von Königinnenfuttersaft, aber nur wenige Untersuchungen, mit denen die oft widersprüchlichen Praktiken zu erklären wären. Aus diesem Grund erschien es mir notwendig, den biologischen Gesetzmäßigkeiten bei der Erzeugung von Weiselfutter im Bienenvolk nachzugehen. Dabei ergab sich eine Reihe eng miteinander verknüpfter Einzelfragen : 1 . Wieviel Futtersaft produziert ein entweiseltes Pflegevolk in mehrmals aufeinanderfolgenden Pflegeansätzen ? 2. In welchem Maße verändert sich die pro Zelle abgelagerte Futtersaftmenge mit fortschreitender Pflegezeit? 3. Beeinflußt die rückläufige Futtersaftablagerung bei wiederholter Pflege das Larvenwachstum? 4. Gibt es eine Beziehung zwischen Futtersaftmengen und Larvengewichten innerhalb der Pflegeserien? 5. Hat das Alter der Arbeiterlarven beim Umbetten in die Weiselbecher einen Einfluß auf die später zu erntende Futtersaftmenge? 6. Welchen Einfluß hat die Beschaffenheit der Weiselbecher auf ihre Bevorratung mit Futtersaft? Aus der Beantwortung dieser Fragen werden Schlußfolgerungen für die praktische Gewinnung von Königinnenfuttersaft erwartet. 


\section{METHODIK}

Um nachvollziehbare Verhältnisse zu schaffen, verwendete ich zu meinen Versuchen stets das gleiche Verfahren der Weiselpflege. Ich benützte entweiselte Völker, deren Königinnen 9 Tage vor Zuchtbeginn in den Honigraum abgesperrt worden waren. Nach Entfernen der Stockmutter und der offenen Brut wurde das Volk, das zuvor in der Regel 27 Waben belagerte auf 9 Zanderwaben $(20 \times 40 \mathrm{~cm})$ zusammengedrängt. Es behielt alle Bienen und einen Teil der gedeckelten Brut. Ich achtete auf gute Pollenvorräte und Versorgung mit genügend Kohlenhydratfutter. In jedem Pflegevolk wurden in bestimmter Zeitabfolge mehrere Pflegeansätze durchgeführt. Dabei erhielt das Volk weder Verstärkung durch Bienen und Brut noch Futter.

Zur Gewinnung altersmäßig genau datierten Umlarvmaterials setzte ich Königinnen für ca. 12 Stunden unter Absperrgitter auf leere Waben.

Bei der Futtersaftentnahme aus den Zellen entfernte ich zunächst die Made mit Hilfe einer Federstahlpinzette (Abb. 1). Der Futtersaft wurde mit einem handelsüblichen Futtersaftspatel herausgeholt und nach Abstreifen in ein Gläschen auf $1 \mathrm{mg}$ genau gewogen. In der Regel ermittelte ich auch das Gewicht der dazugehörigen Maden. Zu diesem Zweck wurden die Maden vorsichtig auf einem Stück Filterpapier vom anhaftenden Futtersaft befreit und auf $1 / 10 \mathrm{mg}$ genau gewogen.

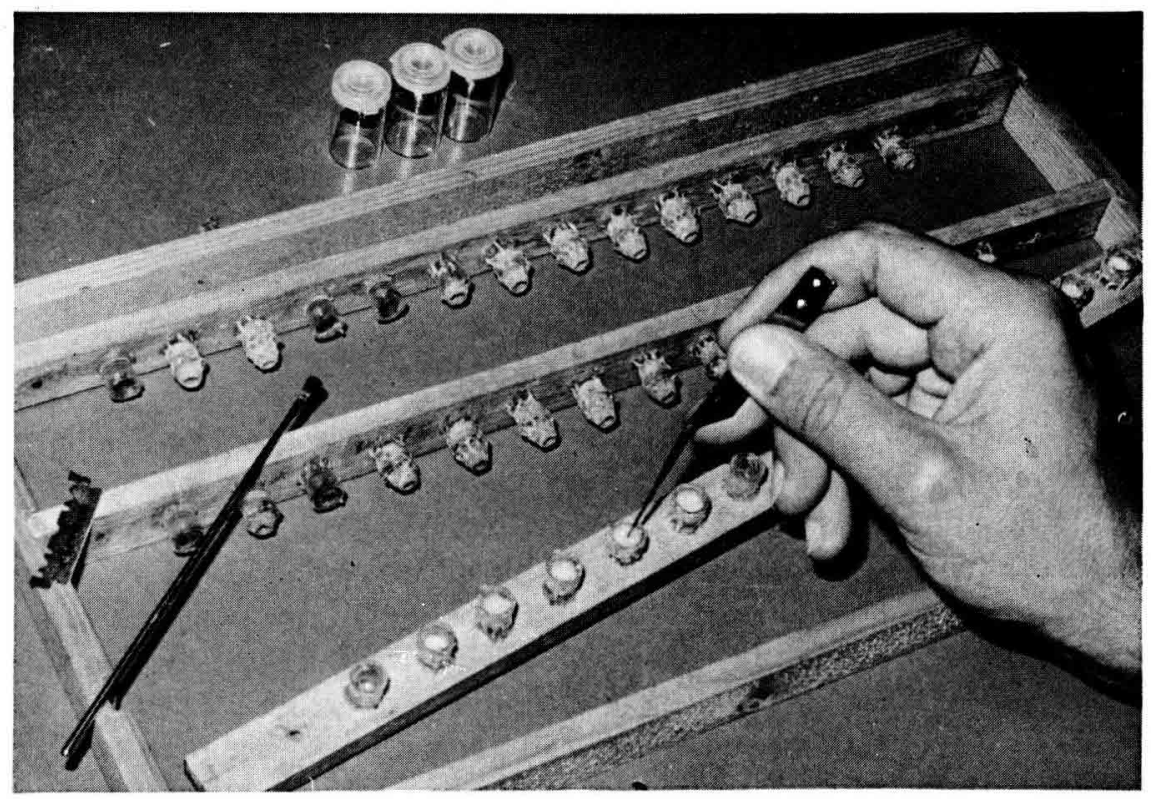

Авв. 1. - Die Maden werden mit der Federstahlpinzette aus den Zellen entfernt, ehe der Futtersaft mit dem Spatel (links im Bild) herausgeholt wird.

Die halbe Rasierklinge dient zum Abschneiden der Zellaufbauten bis zum Rand der Kunststoffnäpfchen. Der Futtersatt wird in Gläschen mit Schnappdeckel gesammelt.

Fig. 1. - Larvae are removed from the cells with a pair of tweezers. The royal jelly is then removed.

The razorblade on the left serves to cut the cell off to the rim of the plastic cell cup.

The royal jelly is weighted after it is collected in vials with snap cover. 
Ich arbeitete ausschließlich mit Carnica-Völkern, sowohl als Spender für den Zuchtstoff als auch zum Zwecke der Weiselpflege. Die Versuche liefen ab dem letzten Julidrittel und im August bei mäßiger Tracht.

\section{ERGEBNISSE}

\section{Futtersafterzeugung eines entweiselten Volkes bei wiederholter Pflege}

Nach SмIтн (1959) ist mit der größten Menge abgelagerten Futtersaftes in den Weiselzellen zu rechnen, wenn die jungen, in die Weiselbecher umgebetteten Arbeiterlarven nach 3 Tagen Pflege als Königinnenlarve ca. 4 Tage alt geworden sind. Somit bietet sich in einem weisellosen Pflegevolk ein 3-Tageturnus zur Futtersaftgewinnung an. Ich entnahm den Futtersaft in Abständen von 3 Tagen aus den angebrüteten Zellen und setzte gleichzeitig einen neuen Zuchtrahmen mit frisch umgebetteten Larven ein. Das Ergebnis dieses Versuches ist in Tabelle 1 wiedergegeben. Die Futtersaftmenge ging im Verlauf von 7 aufeinanderfolgenden Plegeansätzen von $15,7 \mathrm{~g}$ auf $1,3 \mathrm{~g}$ (also etwa auf $1 / 12$ ) zurück und das durchschnittliche Futtersaftgewicht pro Zelle fiel von $562 \mathrm{mg}$ auf $333 \mathrm{mg}$. Das geschah, obgleich bis zuletzt gedeckelte Brut vorhanden war und laufend Jungbienen schlüpften. Am Schluß der Pflegefolge - das war nach 21 Pflegetagen - hatte dieses Volk insgesamt 53,4 g Weiselfuttersaft geliefert. In einem anderen Fall erntete ich bei gleicher Versuchsanordnung 41,6 g und in einem dritten nur 30,0 g Weiselfuttersaft. Im zweiten Fall war die gewonnene Futtersaftmenge der II. Pflegeserie bei einer Annahme von 29 Zellen - gegenüber 13 Zellen in der ersten - größer als in der I. Serie. Das dritte, am wenigsten ergiebige Pflegevolk nahm in nahezu allen Serien weniger Zellen an. Andererseits lagerten die Bienen dieses Volkes in der I. Serie nach 3 Tagen Pflegezeit teilweise die enorme Futtersaftmenge von über $700 \mathrm{mg}$ pro Zelle ab.

\section{Larvenwachstum bei wiederholter Pflege}

In aufeinanderfolgenden Pflegeansätzen verlangsamte sich allmählich die Entwicklung der Larven. Spätestens von der IV. Serie an konnte man das am Deckelungszeitpunkt ablesen, der sich um einen Tag - später noch länger verzögerte. In $\mathrm{Abb} .2$ ist der Gewichtsabfall der ca. 1 tägig umgebetteten Larven nach 3 Tagen Pflegezeit im Verlauf von 6 aufeinanderfolgenden Pflegeserien graphisch dargestellt. Bei einer bereits bei der I. Serie vorhandenen sehr hohen Streuung nimmt das Durchschnittsgewicht der ca. 4 tägigen Maden nach dem III. Pflegeansatz erheblich ab. Im V. und VI. Pflegeansatz gibt es Tiere, die nach 3 Tagen Pflege in einer Weiselzelle so gut wie überhaupt nicht gewachsen sind. 
TAB. 1, - Futtersafiernten in sieben aufeinanderfolgenden Pflegeserien in einem weisellosen Pflegevolk $(A)$

TABL. 1. - Harvested royal jelly in seven consecutive nursing series in a queenless colony (A)

\begin{tabular}{|c|c|c|c|c|c|c|}
\hline \multirow{2}{*}{$\begin{array}{l}\text { Pflegevolk } \\
\text { u. Pflege- } \\
\text { folge } \\
\text { Nurse col. } \\
+ \text { series }\end{array}$} & \multirow{2}{*}{$\begin{array}{l}\text { Pflegezeit } \\
\text { in Tagen } \\
\text { Nursing } \\
\text { (days) }\end{array}$} & \multicolumn{2}{|c|}{$\begin{array}{l}\text { Zahl der Weiselwiegen } \\
\text { Number of cell cups }\end{array}$} & \multicolumn{2}{|c|}{$\begin{array}{l}\text { Futtersaftmenge (mg)/Zelle } \\
\text { Royal jelly }(\mathrm{mg}) / \text { cell }\end{array}$} & \multirow{2}{*}{$\begin{array}{c}\begin{array}{c}\text { Futtersaft- } \\
\text { menge (g) } \\
\text { insges. }\end{array} \\
\text { Total royal jelly } \\
\text { (g) }\end{array}$} \\
\hline & & $\begin{array}{c}\text { gegeben } \\
\text { given }\end{array}$ & $\begin{array}{l}\text { ange- } \\
\text { nommen } \\
\text { accepted }\end{array}$ & $\begin{array}{l}\overline{\mathrm{x}} \pm s \\
\overline{\mathrm{x}} \pm \mathrm{s}\end{array}$ & $\begin{array}{l}\text { Min-Max } \\
\text { min-max }\end{array}$ & \\
\hline A & 3 & 42 & 28 & $562 \pm 60,9$ & $431-654$ & 15,7 \\
\hline II & 3 & 42 & 33 & $308 \pm 72,7$ & $158-414$ & 10,2 \\
\hline III & 3 & 42 & 25 & $395 \pm 101,1$ & $190-512$ & 9,9 \\
\hline IV & 3 & 28 & 20 & $371 \pm 64,6$ & $212-473$ & 7,4 \\
\hline V & 3 & 28 & 21 & $273 \pm 50,6$ & $150-401$ & 5,7 \\
\hline VI & 3 & 28 & 11 & $291 \pm 95,1$ & $147-425$ & 3,2 \\
\hline VII & 3 & 28 & 4 & $333 \pm 89,7$ & $212-429$ & 1,3 \\
\hline sa & & 238 & 142 & & & 53,4 \\
\hline
\end{tabular}

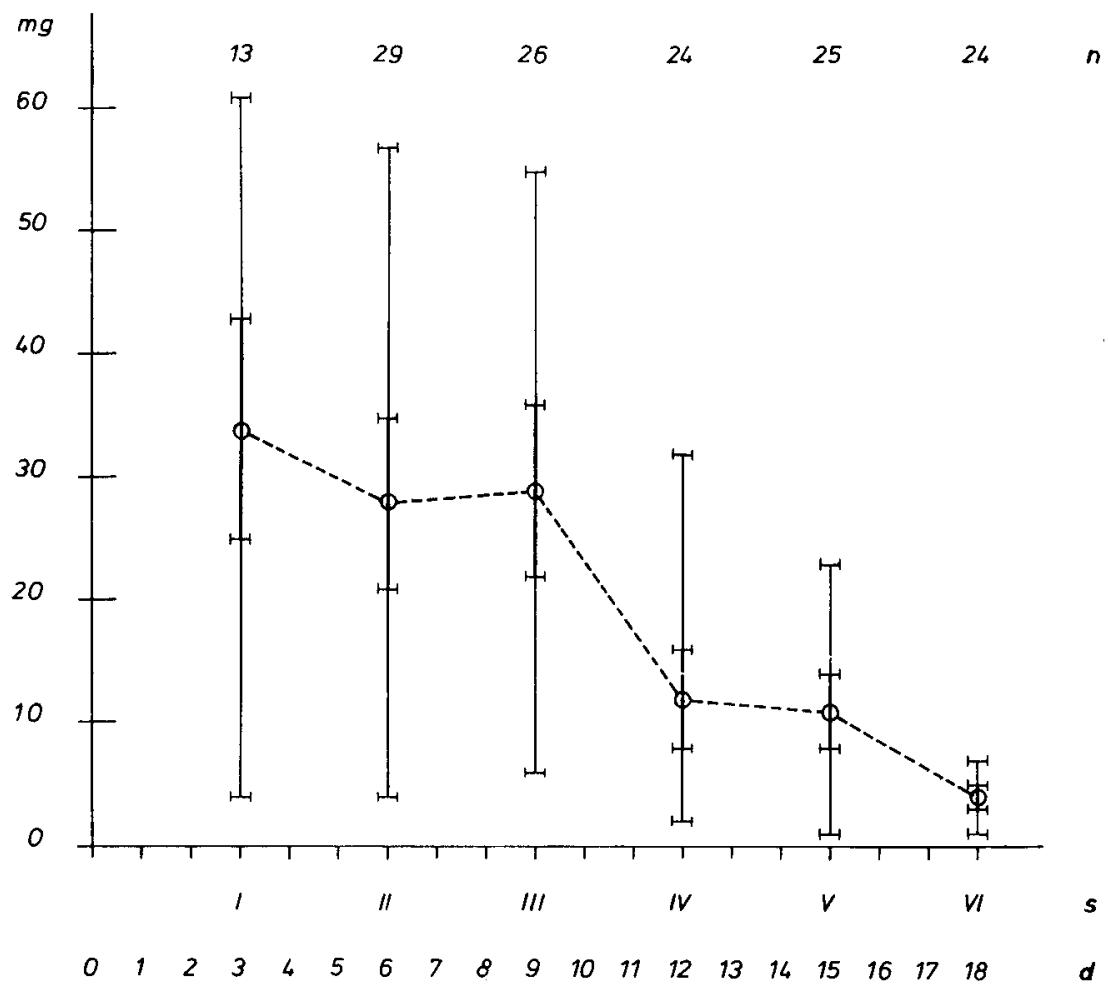

Aвв. 2. - Veränderung der durchschnittlichen Larvengewichte

(mit Standardabweichung u. Variationsbreite) im Verlauf von 6 aufeinanderfolgenden, je 3 Tage dauernden Pflegeserien (s) in einem weisellosen Pflegevolk $\mathbf{n}=$ Zahl der angenommenen Zellen, $\mathrm{d}=$ Versuchsdauer in Tagen

FIG. 2. - Variation of larval weight (average, standard deviation and range) during 6 consecutive series (s) with 3 nursing days for each in a queenless colony $(\mathrm{n}=$ number of accepted cell cups, $\mathrm{d}=$ days of the experiment) 


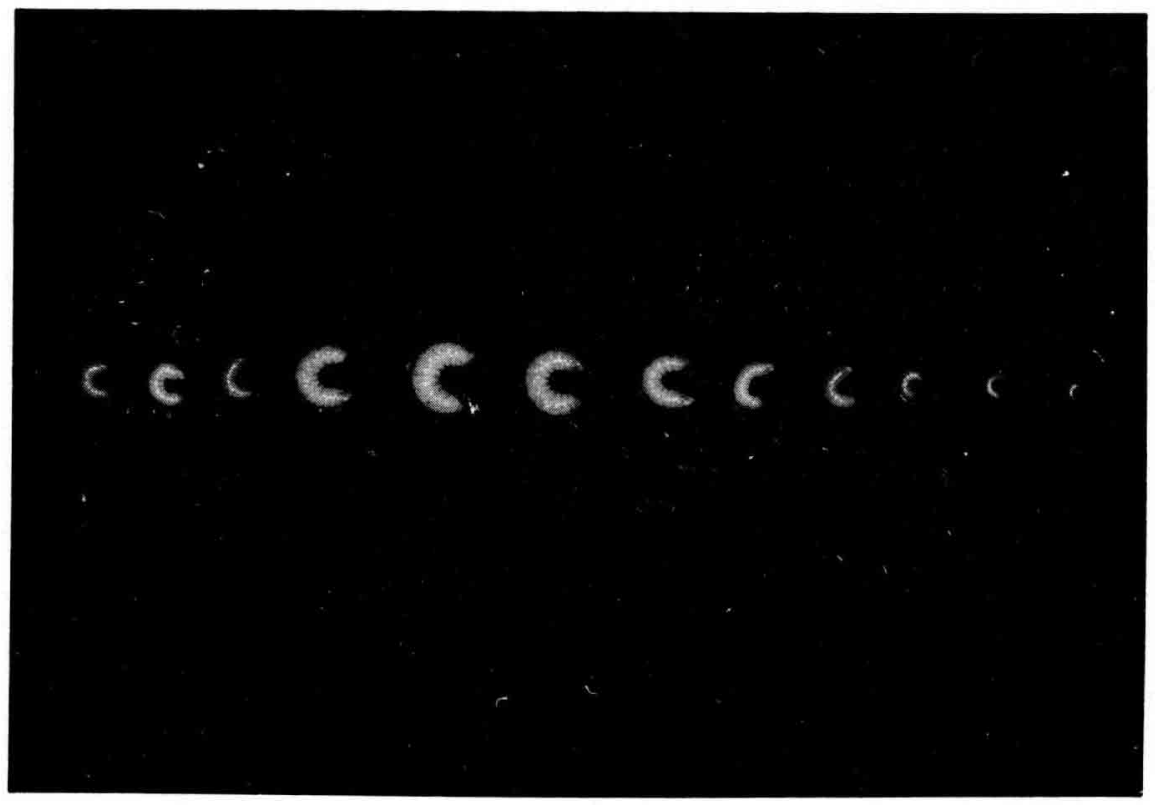

Авв. 3. - Größenverteilung der Larven auf einer Zuchtlatte nach 4 tägiger.Weiselpflege im V. Pflegeansatz

FIG. 3. - Distribution of larvae according to larval size on the ledge of a grafting frame after 4 days of nursing in the fifth series

Mit dem Altern des Pflegevoikes kann man bei den späteren Pflegeansätzen mitunter ein Gewichtsgefälie der Maden nach beiden Außenseiten des Zuchtrahmens feststellen. Für Abb. 3 habe ich aus dem V. Ansatz einer Pflegefolge die Maden aus den Weiselbechern einer der beiden Zuchtlatten des Zuchtrahmens der Reihe nach aufgelegt. Sie befanden sich bis dahin 4 Tage in den Weiselzellen.

\section{Pflegedauer und Futtersaftablagerung}

Die Wachstumsverzögerung der Larven im Laufe wiederholter Pflegeansätze im weisellosen Volk läßt die Frage nach dem Zeitpunkt der größten Futtersaftansammlung in den Weiselzellen in neuem Licht erscheinen. Nach SMITH (1959) nimmt die Larve zwischen ihrem 4. und 5. Lebenstag mehr Nahrung zu sich, als die Ammenbienen nachzufüttern vermögen. Das gilt aber offenbar nur im frisch entweiselten Pflegevolk. In Tabelle 2 werden die durchschnittlichen Futtersaftmengen in den Weiselzellen nach verschieden langer Pflegedauer in der I. und in der IV. und V. Pflegeserie miteinander verglichen. Bei diesem Versuch entnahm ich nach jedem Pflegetag einen Teil der insgesamt angenommenen Zellen zur Bestimmung 


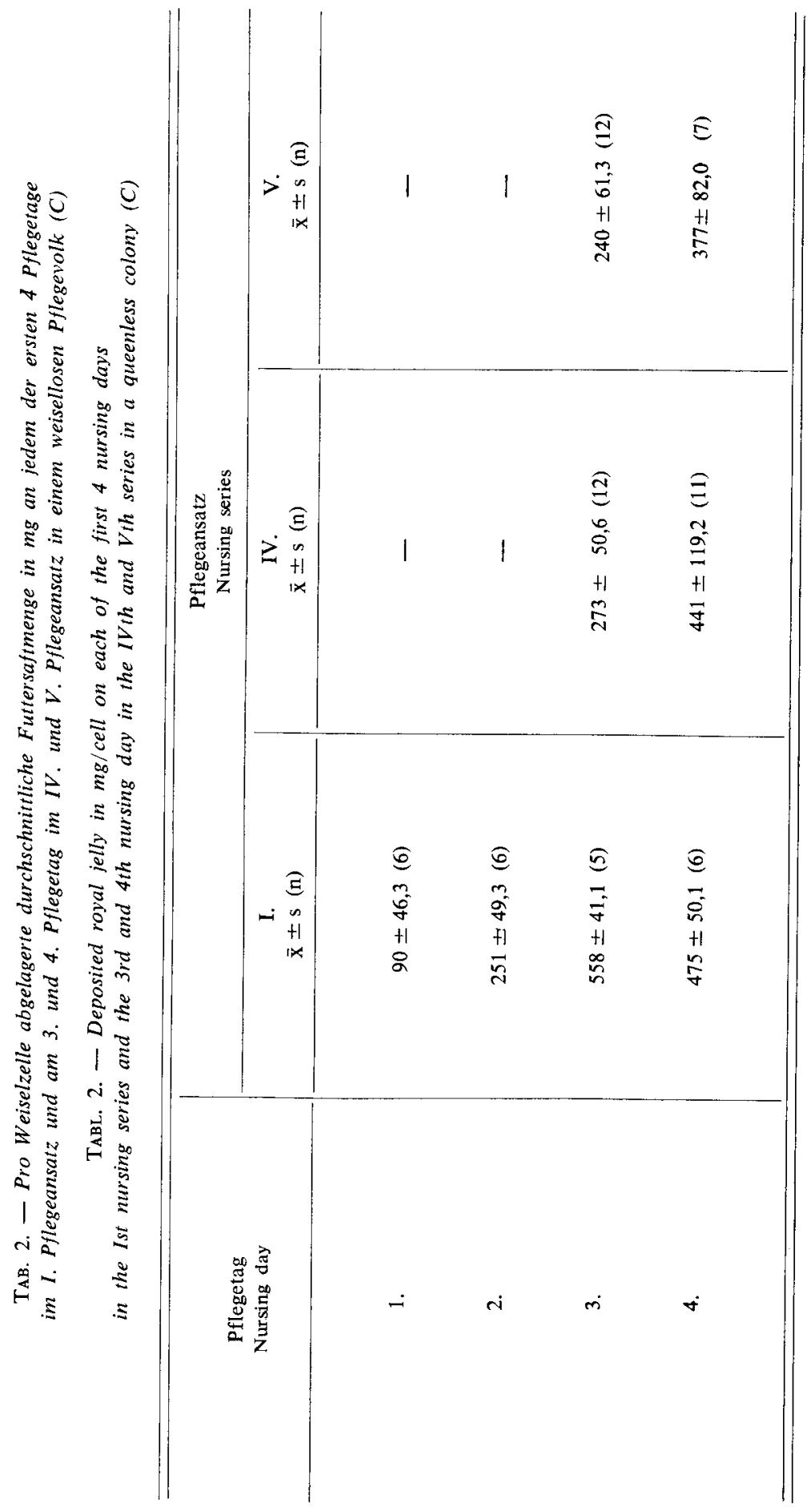




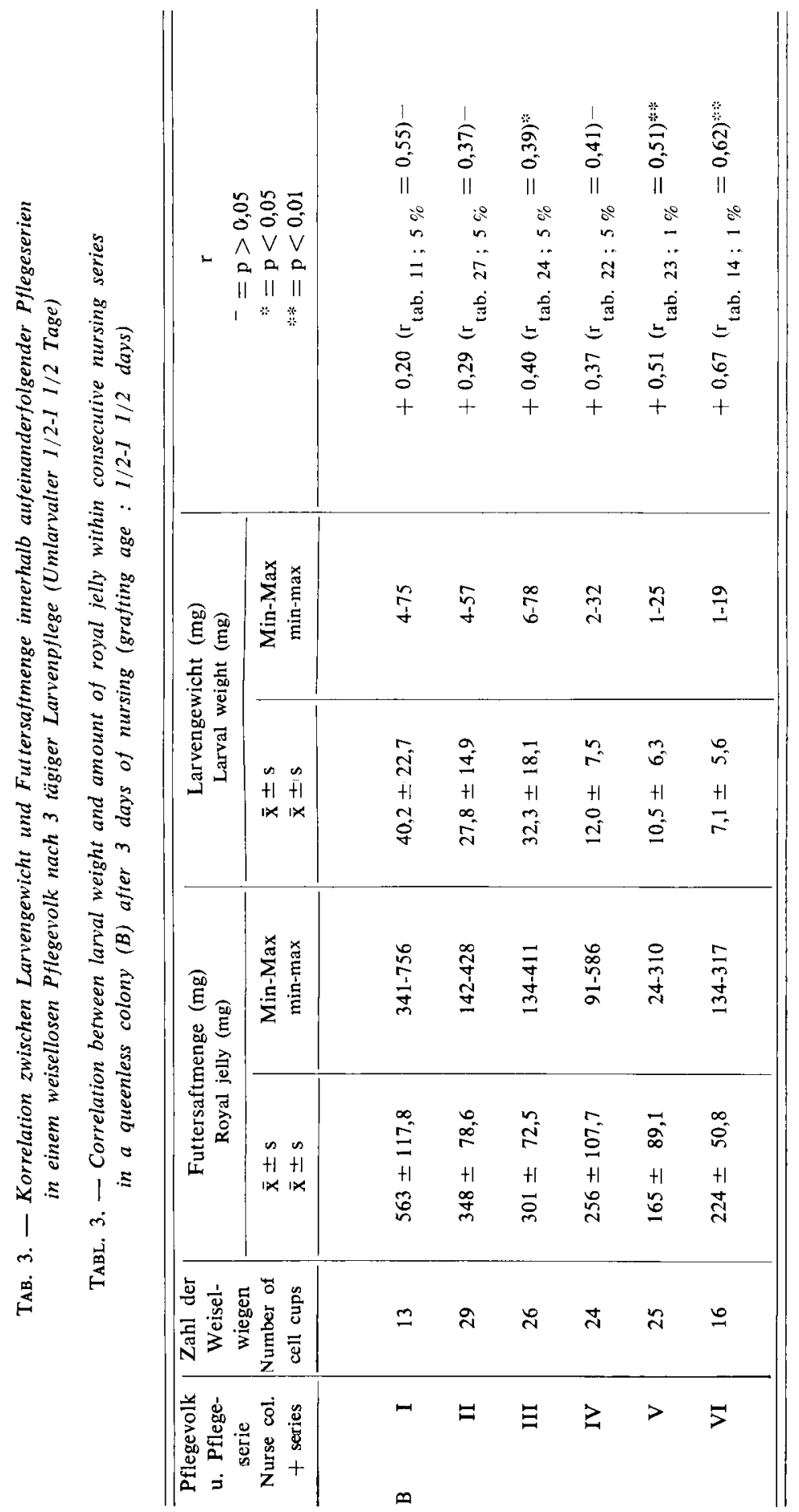


der Futtersaftmengen und gab dieselbe Anzahl gleichaltriger Zellen aus einem anderen Pflegevolk zurück. So versuchte ich, einer Überfütterung der jeweils übrigbleibenden restlichen Maden zu begegnen. In der I. Pflegeserie steigt die abgelagerte Futtersaftmenge erwartungsgemäß bis zum 3. Pflegetag an und nimmt am 4. Tag wieder ab. Zu dieser Zeit waren bereits 3 der untersuchten 6 Zellen gedeckelt. Dagegen nahmen in der IV. und V. Pflegeserie die durchschnittlichen Futtersaftmengen auch vom 3 . auf den 4 . Pflegetag noch einmal deutlich zu, und es gab noch keine Deckelung.

\section{Larvengewicht und Futtersaftmenge}

Ungeachtet der Tatsache, daß die Futtersaftmenge wie auch das durchschnittliche Larvengewicht in einem wiederholt zur Pflege verwendeten Volk mit fortschreitender Serienzahl abnehmen, stellt sich die Frage nach einer Beziehung zwischen Larvengewicht und dazugehöriger Futtersaftmenge innerhalb der Serien. Ich habe in einer Pflegefolge mit 6 Pflegeansätzen die Korrelationen berechnet (Tabelle 3). Bei der hohen Streuung sowohl der Larven- als auch der Futtersaftgewichte war zumindest innerhalb der ersten Pflegeserien keine Korrelation feststellbar. Eine solche trat erst ab der III. Serie in Erscheinung.

\section{Alter der Maden beim Umlarven und Futtersaftablagerung}

Zur Frage, ob das Alter der Maden beim Umlarven einen Einfluß auf die nach 3 Tagen in den Zellen abgelagerte Futtersaftmenge ausübt, belarvte ich Näpfchen an einem Zuchtrahmen in abwechselnder Reihenfolge mit 0-1/2, 1/2-1 und 1-1 1/2 Tage alten Maden. Für den Versuch benützte ich den I. und II. Pflegeansatz in einem entweiselten Pflegevolk. Wie aus Tabelle 4 hervorgeht, schwanken die nach 3 Pflegetagen pro Zelle abgelagerten durchschnittlichen Futtersaftmengen willkürlich. Das bedeutet, daß die Bienen den Futtersaft - zumindest innerhalb des untersuchten Altersspielraums — nicht in Abhängigkeit vom Alter der Maden ablagern. Für die erzeugte Futtersaftmenge scheint somit allein die Zeitdauer der Larvenpflege maßgeblich zu sein.

\section{Wachs- und Kunststoffbecher}

In mehreren Völkern und in wiederholten Pflegeansätzen verglich ich wechselweise am gleichen Zuchtrahmen befestigte Weiselbecher aus Wachs und aus Kunststoff hinsichtlich der abgelagerten Futtersaftmengen. Die Wachsbecher waren nach dem Tauchverfahren mit $9 \mathrm{~mm}$ starken Formhölzern hergestellt, die Kunststoffbecher bestanden aus Polystyrol. Ich verwendete zuerst ein deutsches, konisch geformtes Fabrikat mit ebenem Innenboden, später nahm ich zylindrische, haupt- 
TAB. 4. - Pro Zelle abgelagerte Futtersaftmengen 3 Tage nach dem Umlarven von $0-1 / 2,1 / 2-1$ und 1-1 1/2 tägigen Maden im I. und II. Pflegeansatz eines weisellosen Pflegevolkes (D)

TABL. 4. - Deposited royal jelly/cell 3 days after grafting larvae $0-1 / 2$, I/2-1 and 1-1 1/2 days old in the Ist and IInd series in a queenless colony (D)

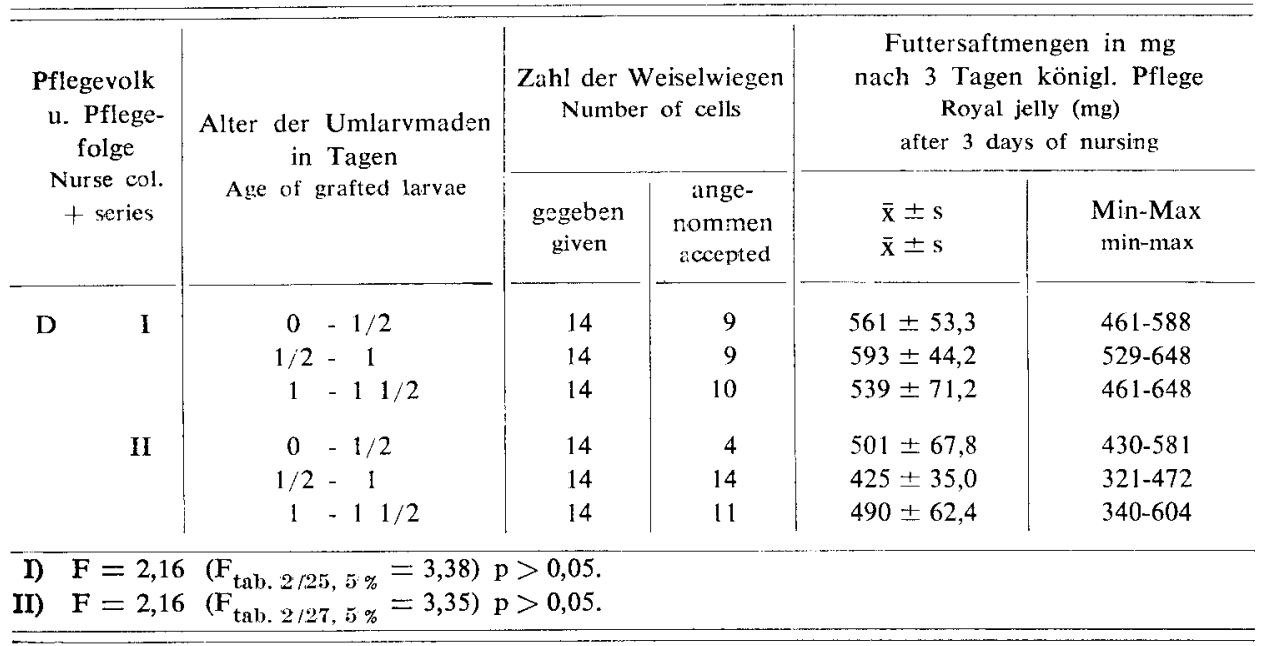

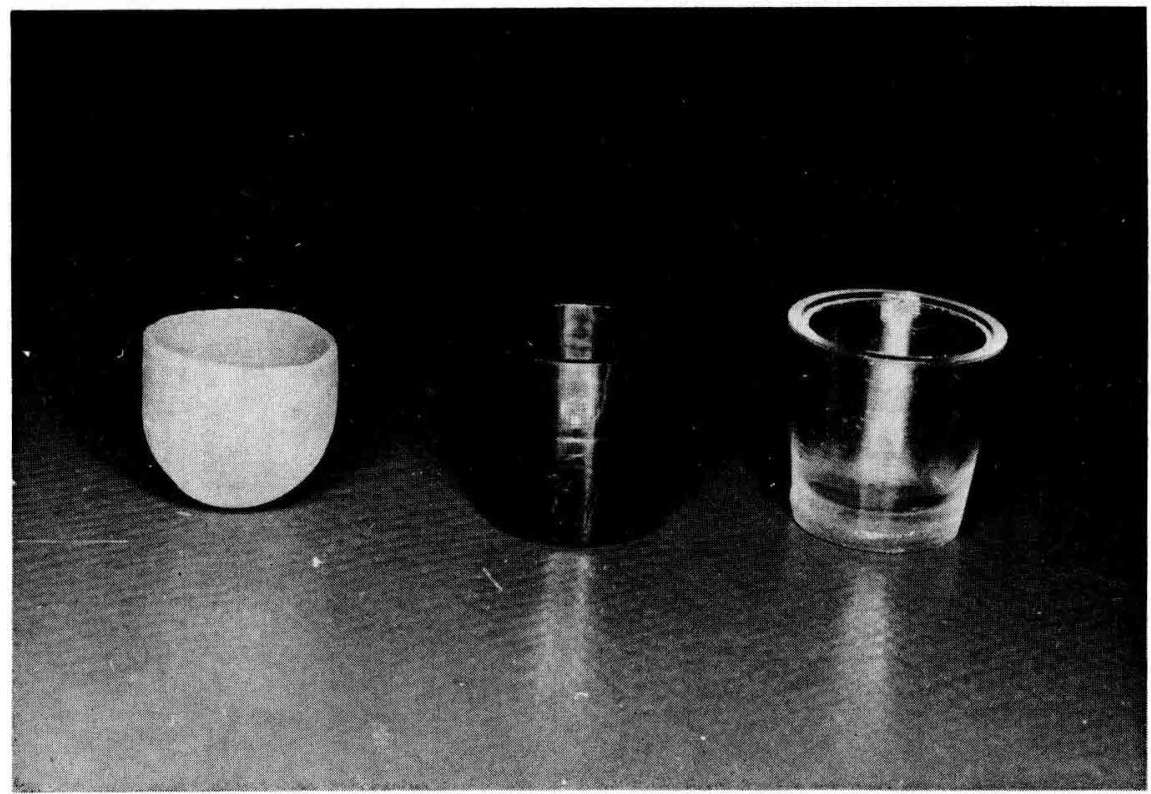

Авв. 4. - Neben «getauchten» Wachsnäpfchen warden zwei unterschiedliche Formtypen von Polystyrolbechern zu den Vergleichsversuchen über die Ablagerungsmengen des Weiselfuttersaftes verwendet FIG. 4. - Three types of cups (wax, on the left, and plastic) used in a comparative experiment for royal jelly production 

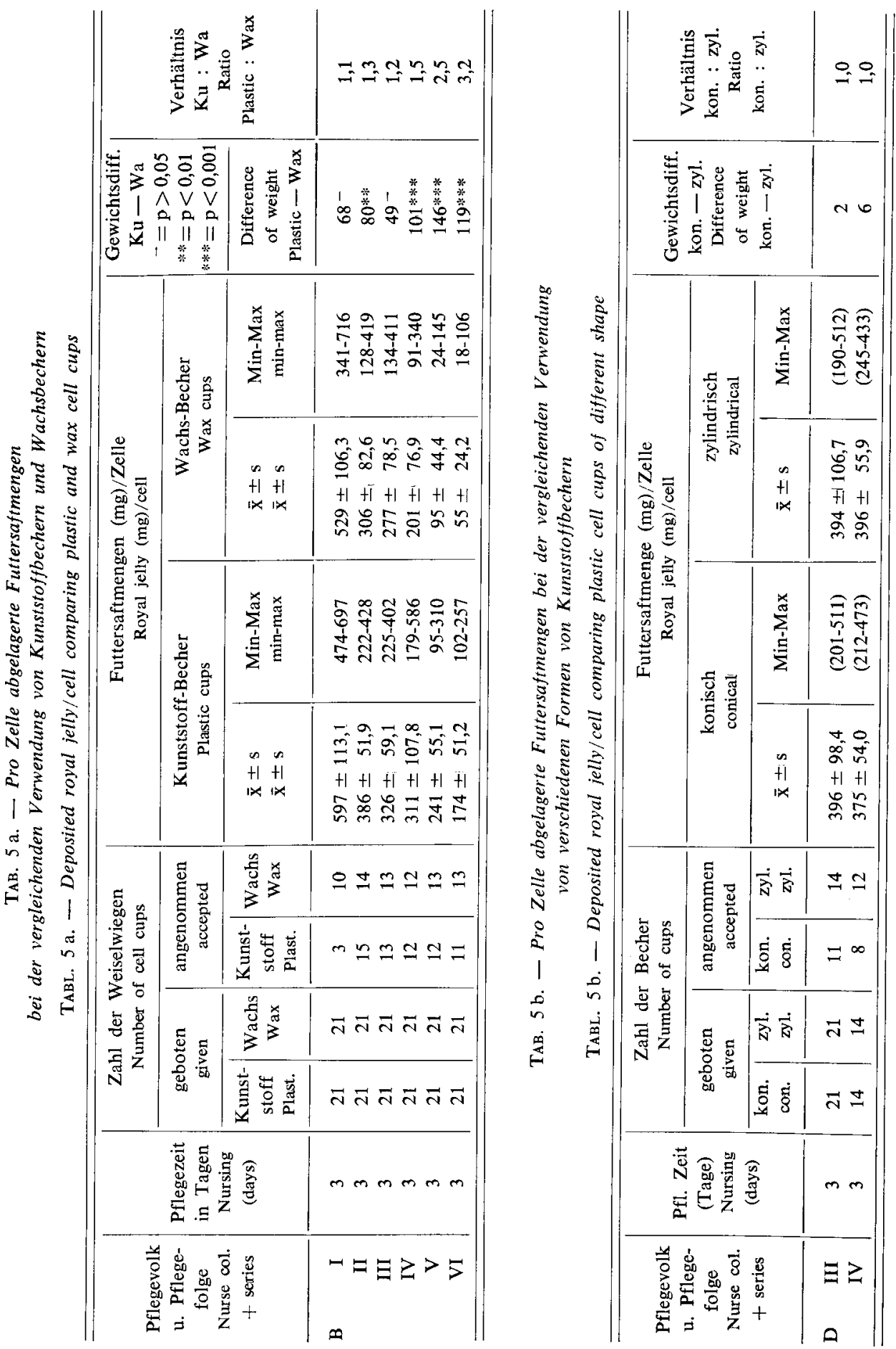
sächlich in Frankreich verbreitete Becher mit rundem inneren Boden dazu (Abb. 4). Sowohl Wachs- als auch Kunststoffnäpfchen wurden wiederholt zur Futtersaftgewinnung verwendet. Ich unterzog die ausgekratzten Näpfchen vor ihrem neuerlichen Gebrauch keiner zusätzlichen Reinigung, da ich in Wahlversuchen feststellen konnte, daß die Bienen frische Wachs- bzw. fabrikneue Kunststoffbecher ebensogut annehmen und mit Futtersaft versorgen wie bereits benutzte. Umgebettet wurden ca. 1 tägige Maden. Im Beispiel einer Pflegefolge mit 6 in Abständen von 3 Tagen durchgeführten Pflegeansätzen (Tabelle 5 a) vergrößerte sich die Differenz zwischen den Futtersaftmengen in den Wachs- und in den (konischen) Kunststoffbehältern mit jeder neuen Serie zugunsten der Kunststoffbehälter. Das Verhältnis der Futtermenge von Kunststoffbecher : Wachsbecher stieg kontinuierlich von 1,1 in der I. Serie auf 3,2 in der VI. Serie. Das bedeutet, daß in der VI. Serie die Kunststoffbecher mehr als dreimal so viel Futtersaft enthielten wie die Wachsbecher. Besonders in den späteren Serien ist der Unterschied zwischen den beiden Becherarten hoch gesichert. Beim Vergleich der konischen mit den zylindrischen Kunststoffbehältern in einem anderen Pflegevolk konnte ich dagegen (auf fortgeschrittener Pflegefolge) keinen Unterschied in den abgelagerten Futtersaftmengen feststellen (Tab. 5 b).

\section{DISKUSSION}

$\mathrm{Zu}$ 1.) Die Beobachtung, daß der augenfällige Rückgang der Futtersafterzeugung im entweiselten Volk von Pfiegeserie zu Pflegeserie gelegentlich erst nach der II. Serie einsetzt, haben schon Mirza und Parac (1961) gemacht. Auch weiß der Königinnenzüchter, daß Annahme und Versorgung der Königinnenmaden oft erst in der II. Zuchtserie auf voller Höhe sind. Obgleich in den Nachfolgezuchten die Futtersaftüberschüsse allmählich zurückgehen, lassen sich trotzdem noch in mehreren Serien vollwertige Königinnen erzielen (WEISS, 1972).

Die bei den Versuchen angefallenen Futtersafternten wären wahrscheinlich höher ausgefallen, wenn die Versuche nicht im ausgehenden Sommer, sondern früher im Jahr durchgeführt worden wären. Wava und HaNNA (1967) erzielten die größten Futtersafternten im «Frühling ».

$\mathrm{Zu}$ 2.) Die Verzögerung des Larvenwachstums in gealterten Pflegevölkern ist aus Versuchen mit wiederholter Königinnenerzeugung im « unverjüngten Pflegevolk» bereits bekannt (GoetZe, 1924, 1926 ; Buchner, 1953 ; WeIss, 1972). Vielleicht hat die von Goetze (1924) und BUCHNER (1953) konstatierte sinkende Temperatur im alternden Pflegevolk etwas damit zu tun, was auch erklären könnte, daß bei älteren Pflegeserien die Maden in der Mitte des Zuchtrahmens besser wuchsen als die seitlich gelegenen. Dagegen spricht aber die nach 15 Pflegetagen unveränderte Stärke des Pflegevolkes. Die verzögerte Larvenentwicklung 
kann auch nicht auf Nahrungsmangel zurückgeführt werden, denn trotz rückläufiger Futtersaftablagerung hatten die Larven stets einen Futterüberschuß. Eher möchte man annehmen, daß sich der Futtersaft bei starker Beanspruchung der Pflegebienen bzw. bei deren Älterwerden qualitativ vom Futtersaft des frisch entweiselten Volkes unterscheidet.

$\mathrm{Zu}$ 3.) $\mathrm{Da}$ mit fortschreitender Pflegefolge spätestens ab der III. Serie die. Futtersaftmenge in den Zellen auch am 4. Pflegetag und sogar später noch zunimmt, ohne daß die Zellen gedeckelt werden, kann bei der Futtersaftgewinnung ein entsprechender Aufschub der Futtersaftentnahme zu einem besseren Ernteergebnis führen. Wenn in meinen Versuchen (im I. Pflegeansatz) die täglich pro Zeile abgelagerten durchschnittlichen Futtersaftmengen (s. Tabelle 2) durchwegs, höher lagen als die entsprechenden Werte von SмIтH, 1959 (2. Tag $147 \mathrm{mg}$, 3. Tag $235 \mathrm{mg}, 4$. Tag $182 \mathrm{mg}$ ) und Okada und Obata, 1963 (1. Tag 79,1 mg, 2. Tag. $244,4 \mathrm{mg}, 3$. Tag 40:0,2 mg) mögen dafür die Art cies Pflegeverfahrens und die Zahl der gebotenen und angenommenen Weiselzellen verantwortlich sein, aber auch Gründe, wie Bienenrasse, Beschaffenheit der Weiselbecher, Jahreszeit der Futtersaftgew:nnung $\mathbf{u}$. dgl. sind denkbar.

$\mathrm{Zu}$ 4.) Das Fehlen einer Korrelation zwischen dem Larvengewicht und dem Gewicht der zugeordneten Futtersaftmenge, zumindest innerhalb der ersten Pflegeserien, kann nicht sonderlich überraschen, wenn man weiß, daß auch zwischen dem Gewicht der Königinnenpuppen kurz vor dem Schlupf und den in den zugehörigen Zellen befindlichen Restfuttermengen - nicht nur in derersten, sondern auch in späteren Serien - keinerlei Beziehung besteht. Das er-. brachte die Auswertung des umfangreichen Zahlenmaterials meiner Versuche mit. aufeinanderfolgenden Zuchten in unverjüngten Pflegevölkern (WeIss, 1972).

$\mathrm{Zu}$ 5.) Hinweise, daß die Bienen den Königinnenfuttersaft unabhängig vom Alter der umgelarvten Maden ablagern (in meinen Versuchen zumindest innerhalb. des Altersspielraumes von 1/2-1 1/2 Tagen), findet man bereits bei WAVA und HanNa (1967) und Chang (1979). Da für die abgelagerte Futtersaftmenge offenbar allein die Zeitdauer der königlichen Larvenpflege maßgebend ist, wird die Spekulation hinfällig, durch Umlarven älterer Maden und Verkürzung der Pflegedauer die Futtersafterzeugung zu rätionalisieren.

$\mathrm{Zu}$ 6.) Wachsbecher und Becher aus Polystyrol werden gleich gut von den Bienen angenommen (Smith, 1959 ; InOUE und Inoue, 1963 ; WaFA und Hanna, 1967; WeIss u. M., 1976). Wenn einige Autoren (WaFA und HanNa, 1967; Chang, 1979, Manino und Marletto, 1981) den in meinen Versuchen so auffälligen Unterschied in der Futtersaftablagerung zugunsten der Kunststoffbecher nicht bemerkt haben, kann das daran liegen, daß nicht mit wiederholten Pflegeansätzen gearbeitet wurde, oder daß Besonderheiten des Materials, aus dem die Becher bestanden, mitspielten. Die Frage, ob ein Volk das nicht wahlweise Wachs-. 
und Kunststoffbecher, sondern ausschließlich Kunststoffbecher bekommt, mehr Futtersaft produziert als ein Volk nur mit Wachsbehältern, läßt sich experimentell nicht einwandfrei nachprüfen. Aber zahlre:che Versuche, in denen ich im Ablauf einer Pflegefolge nacheinander entweder nur mit Wachs- oder nur mit Kunststoffbechern arbeitete, sprechen für diese Annahme.

Warum die Kunststoffbecher besser mit Weiselfuttersaft versorgt werden als Wachsnäpfchen, hängt offenbar nicht mit äußeren oder inneren Formkriterien der Becher zusammen. Beispielsweise hatten die zylindrischen Becher in meinem Versuch Hohlböden, die Böden der konischen Becher waren flach. In früheren Versuchen mit Wachsbechern hatte ich entgegen Vuillaume (1957 a) bereits festgestellt, daß die Ausformung des Bodens für die Larven-Annahme bedeutungslos ist (Weiss et al., 1979). Der innere Durchmesser der Becher, der nach Vuillaume (1957b, 1957c) die Futtersaftablagerung beeinflussen soll, kann hier ebenfalls keine Rolle gespielt haben, er betrug bei allen Bechern $9^{\prime} \mathrm{mm}$. Auch die Größe des Becherinnenraums liefert keine Erklärung für die unterschiedliche Futtersaftversorgung der Kunststoff- und Wachsbehälter. Die Bechervolumen betrugen $49 \mathrm{~mm}^{3}$ (Kunststoffbecher, konisch), $45 \mathrm{~mm}^{3}$ (Kunststoffbecher, zylindrisch) und $46 \mathrm{~mm}^{3}$ (Wachsbecher). Indirekt könnte aber doch das Fassungsvermögen der Becher eine Rolle spielen. Gleich nach dem Erscheinen der belarvten Wachsbecher im Volk, beginnen die Bienen deren scharfe Ränder abzustumpfen, wobei die Becher vorübergehend an Tiefe verlieren. Wenn die Becher danach hochgezogen werden, wird möglicherweise ein bestimmter Abstand vom Becherrand zur Larve eingehalten. In den Kunststoffbehältern muß die Made möglicherweise erst mit

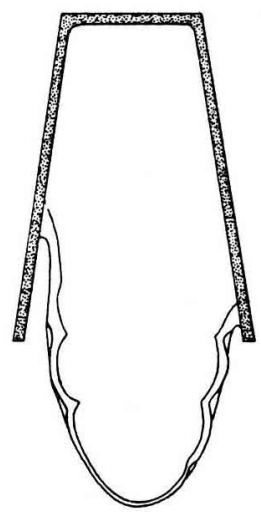

Aвв. 5. - Die Bienen «verbrauchter 》 Pflegevölker beginnen die Weiselzellen oft aus dem Inneren der Kunststoffbecher herauszubauen.

Die Zellen fallen dann wie ihre Insassinnen sehr klein aus

FIG. 5. - In aged nursing colonies bees sometimes begin to build queen cells on the inside of used plastic cups.

Consequently, cells and their occupants are very small 
Hilfe des Futtersaftpolsters gehoben werden, ehe diese Maßarbeit beginnen kann. In älteren Völkern mit versiegenden Futtersaftreserven kann das allerdings nicht mehr funktionieren. Dann setzen die Bienen das erste Wachs zum Ausbau der Weiselzelle in der Regel bereits an der Innenwand der Kunststoffbehälter an (Abb. 5). Die Weiselzellen späterer Zuchtserien fallen damit auch entsprechend kürzer aus.

Während die hier diskutierten Versuchsergebnisse mit Hilfe der Pflege im entweiselten Volk bei mehrfach aufeinanderfolgenden Pflegeansätzen gewonnen wurden, mag man sich, unter dem Eindruck der rasch abnehmenden Futtersaftreserven überlegen, ob nicht anstelle der radikalen Ausschöpfung eines entweiselten Volkes ein anderes Verfahren, etwa die zweigeteilte Pflege im Anbrüte- und Endpflegevolk oder das Pflegeverfahren im weiselrichtigen Volk, besser zur Futtersaftgewinnung geeignet ist. Die meisten kommerziellen Futtersaftproduzenten scheinen denn auch einem solchen Weg den Vorzug zu geben (Lit. bei DadaNT, 1958 ; Vuillaume, 1957 c ; Armbruster, 1960).

Eingegangen im Februar 1985. Angenommen im Januar 1986.

\section{RESUME \\ PRODUCTION DE GELEE ROYALE ET DEVELOPPEMENT DES LARVES ROYALES AU COURS D'EXPERIENCES SUCCESSIVES DANS LES COLONIES ORPHELINES D'APIS MELLIFICA L.}

J'ai étudié la production de gelée royale et la croissance des larves royales dans des colonies orphelines. Dans ce but $j$ 'ai démarré un élevage de reines plusieurs fois de suite dans chaque colonie. Au bout du $3^{\prime \prime}$ ou $4^{\circ}$ jour, j'interrompais l'élevage de reines puis le recommençais. Durant les expériences je n'ai ajouté aux colonies orphelines, ni abeilles, ni couvain. Les abeilles utilisées étaient des carnoliennes (Apis mellifera carnica $\mathbf{L}$.) et les essais ont été menés fin juin et au mois d'août pendant une miellée moyenne à Erlangen (R.F.A.).

1. La quantité de gelée royale récoltée par colonie au bout de 3 jours a diminué à chaque série d'élevage royal. Par exemple dans les premières séries la quantité de gelée royale a été de $15,7 \mathrm{~g}$ et seulement de $1,3 \mathrm{~g}$ dans les $7^{\mathrm{e}}$ séries (tabl. 1). Il s'est passé la même chose dans d'autres expériences. La quantité totale de gelée royale au cours de 7 séries successives dans 3 colonies différentes a été de $53,4 \mathrm{~g}, 41,6 \mathrm{~g}$ et $30,0 \mathrm{~g}$. Au bout de 3 jours les cellules, qui renfermaient des larves greffées à 1 jour, contenaient en moyenne $500 \mathrm{mg}$ de gelée royale dans les premières séries. Certaines cellules contenaient jusqu’à $700 \mathrm{mg}$. La fourniture de gelée royale dépend dans une certaine mesure du nombre de cellules royales acceptées.

2. Le développement des larves greffées a été retardé au cours des expériences successives. Les larves, qui avaient été greffées à l'âge d'1 jour et avaient reçu une nourriture de reine pendant 3 jours, voyaient en moyenne leur poids diminuer à chaque nouvelles séries, malgré la forte variabilité individuelle. Dans les $6^{\circ}$ séries d'un essai, le poids des larves n'a été que le $10^{\circ}$ de celui des premières séries (fig. 2). Certaines larves n'étaient que légèrement plus grosses au bout de 3 jours qu'au moment du greffage. Les larves situées vers les extrémités du cadre de greffage ont vu parfois leurs poids diminuer (fig. 3). La date d'operculation a été repoussée d'un ou plusieurs jours dans les dernières séries. 
3. J'ai trouvé la plus grande quantité de gelée royale dans les cellules 3 jours après le greffage ; ceci est en accord avec les données de la littérature. Mais cela n'a été valable qu'au début des séries successives de tests. A partir des $3^{\text {e }}$ séries, la quantité de gelée royale a été plus élevée le $4^{\mathrm{e}}$ jour (tabl. 2).

4. Il n'y a pas de corrélation entre le poids des larves et la quantité de gelée royale dans les cellules au cours des séries précoces. Plus tard une légère corrélation a été montrée (tabl. 3).

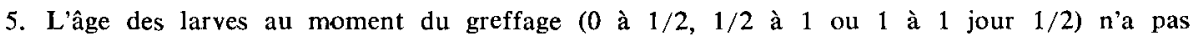
affecté la quantité de gelée royale déposée dans leurs cellules.

6. Dans une série d'expériences, j’ai comparé le dépôt de gelée royale dans des cupules en cire et dans des cupules en polystyrole (fig. 4). On a fixé sur les bords de cadres de greffage une cupule de chaque type alternativement. Les abeilles ont toujours déposé plus de gelée royale dans les cupules en plastique que dans celles en cire au cours des 3 jours d'élevage des larves. Le pourcentage de gelée royale déposée dans les cupules en plastique a augmenté nettement à chaque nouvelle série de tests. Les quantités de gelée royale déposée ont été les mêmes dans les cupules plastiques coniques à fond plat et dans les cupules cylindriques à fond concave (tabl. 5).

Les cupules en plastique peuvent être utilisées aussi souvent que nécessaire mais l'utilisation des cellules en cire est par contre limitée. J'ai testé plusieurs fois des cupules en plastique neuves et usagées en les attachant alternativement sur le même cadre de greffage. Les abeilles ont accepté les cupules neuves ("propres») aussi bien que les usagées («sales») et ont déposé les mêmes quantités de gelée royale.

On peut tirer de ces expériences, des conclusions pour la production commerciale de la gelée royale.

\section{SUMMARY}

\section{DEPOSITION OF ROYAL JELLY AND DEVELOPMENT OF QUEEN LARVAE DURING CONSECUTIVE EXPERIMENTS IN QUEENLESS COLONIES OF APIS MELLIFERA L.}

I investigated the production of royal jelly and the growth of queen larvae in queenless colonies. For this purpose I initiated queen rearing several consecutive times in each colony. After the third or fourth day I interrupted queen rearing and started it again. Neither bees nor brood were added to the queenless colonies during the experiments. I used Carniolan bees and conducted the trials at the end of June and in August during a moderate honey flow in Erlangen (F.R.G.).

1. The quantity of royal jelly gathered per colony at the end of three days became smaller with each successive series in that colony. For example in one colony it totaled $15,7 \mathrm{~g}$ in the first series and diminished to $1,3 \mathrm{~g}$ in the seventh series (table 1). Some other experiments took the same course. The total amont of royal jelly in seven successive series was $53.4 \mathrm{~g}, 41.6 \mathrm{~g}$ and $30.0 \mathrm{~g}$ in three different colonies. After three days cells with larvae grafted when one day old contained an average of about $500 \mathrm{mg}$ royal jelly in the first series. Some cells contained up to $700 \mathrm{mg}$. To some extent the provision of royal jelly depended on the number of queen cells accepted.

2. In successive experiments the development of grafted larvae was delayed. The Iarvae which were grafted one day old and nourished as queens for three days weighed on the average less with each new series, despite the high variability among individual larvae. In the sixth series of one trial, larvae weighed only one tenth as much as in the first series (fig. 2). Some larvae were only slightly larger after 3 days than at the time of grafting. Sometimes larval weight decreased towards the ends of the grafting frame (fig. 3). The date of cell capping was delayed for one day or more during later series. 
3. In accordance with the literature, I found the largest amount of royal jelly in the cells three days after grafting. But this was only true at the beginning of the successive series of tests. From the third series, the amount of royal jelly was higher on the fourth day (table 2).

4. There was no correlation between the weight of larvac and the amount of royal jeliy in the cell in the early series of tests. Later a slight correlation was evident (table 3).

5. The age of larvae at the time of grafting $(0-1 / 2,1 / 2-1$ or $1-11 / 2$ days) did not affect the amount of royal jelly deposited in their cells.

6. In a number of experiments I compared the deposition of royal jelly in queen cell cups of wax with that in cups of polystyrole (fig. 4). Both kinds of cups had been fixed alternately on the edges of grafting frames. In each case the bees added more royal jelly to the plastic cups than to the cups of beeswax during three days of nursing larvae. The ratio of the amounts of royal jelly deposited in each cell type increased clearly in favor of the plastic cups with each new series of tests. There was no difference between the amounts of royal jelly deposited in conical plastic cups with flat bottoms and cylindrical cups with concave bottoms (table 5).

Plastic cups can be used as often as desired but the use of wax cups is limited. I repeatedly tested new and used plastic cups attached alternately to the same grafting frame. The bees accepted the new («clean») cups just as well as the used ("dirty») cups and provided both with the same quantities of royal jelly.

From all the experiments presented in this paper one can make conclusions for the benefit of commercial production of royal jelly in practical beekeeping.

\section{LITERATUR}

Armbruster L., 1960. - Königinnenfuttersaft. Arch. Bienenkd,, 37 (1), 1-39.

BuchNer R., 1953. - Beeinflussung der Größe der Arbeitsbiene durch Raum- und Nahrungsmangel während der Larvenzeit. Roux Arch. Entwickl. mech., 146, 544-579.

Chang S.Y., 1977. - (Effects on size and type of queen cup on the production of royal jelly and acceptance by nurse bees) Master Thesis, National Chung Hsing University, Taichung, Taiwan (chin., engl. Zusf.) AA 30 (1) 201/79 S. 39.

Dadant M.G., 1958, - The production and use of royal jelly. Am. Bee J., 98 (2), 51-52.

Goetze G., 1924. - Einige Versuche zum Einfluß des Alters der Bienen auf die Nachschaffung von Königinnen. Arch. Bienenkd., 6 (5/8), 224-228.

Goetze G., 1926. - Zur Züchtungsbiologie, Variabilitätsstudien an der Honigbiene. Preuß $\beta$. Bienenzeitung (9), 276-283.

Inoue T., Inoue A., 1963. - (Some enquiries into the utilisation of plastic queeen oell cups for royal jelly production). Bee Science, Nagoya, 4 (2), $19-23$ (jap., engl. Zusf.) AA 877/64.

Manino A., Marletto F., 1981. - (Comparison of wax and plastic queencell cups for queen rearing and royal jelly production). Apicoltore Moderno, 72 (6), 217-222 (it., engl. Zusf.) AA 34 (1), 219/83 S. 45.

Mirza E., Barac J., 1981. - (Daten über die Gewinnung des Weiselfuttersaftes) (rum., engl. u. dtsch. Zusf.) Lucrari Stiintifica III., 213-222. 
Okada J., Obata H., 1963. - (Variation in the amount of royal jelly produced at different stages of rearing queens by Doolittle's grafting method). Anim. Husb., 17 (3), 475-477 (jap.) AA 794/65.

Smith M.V., 1959. - The production of royal jelly. Bee World, 40 (10), 250-254.

Vuillaume M., 1957 a. - La forme des cellules royales chez les abeilles. Insectes soc., 4 (4), 385-390.

Vuillaume M., 1957 b. - Contribution à la psychophysiologie de l'élevage des reines chez les abeilles. Insectes soc., 4 (2), $11-156$.

Vujllaume M., 1957 c. - Elevage des reines. Production de gelée royale. Apiculteur, 101 (4), 113-116.

Vuillaume M., 1959. - Les productions simultanées de miel, gelée royale et abeilles sont-elles incompatibles? Ann. Abeille, 4, 271-275.

WaFA A.K., HANNa M.A., 1967. - Einige Faktoren, die die Gewinnung von Weiselfuttersaft beeinflussen. XXI. Internationaler Bienenzüchterkongreß in Maryland. Ref. 22 (dtsch. Fass.) S. $525 / 26$.

WEISS K., 1972. — Verlauf und Beschaffenheit aufeinanderfolgender Zuchtserien im unverjiingten Pflegevolk. Apiacta, VII (3), 110-114/140.

Weiss K., Böttcher F.K., Mautz D., 1976. - Die Tätigkeit der Bayerischen Landesanstalt für Bienenzucht Erlangen im Jahre 1975. Imkerfreund, 31 (3), 66-76

Weiss K., Mautz D., Schaper F., 1979. - Die Tätigkeit der Bayerischen Landesanstalt für Bienenzucht Erlangen im Jahre 1978. Imkerfreund, 34 (3), I-XX. 